\title{
Medical Students' Insights Towards Patient Safety
}

"Saeed Alshahrani, ${ }^{1,2}$ Ahmad Alswaidan, ${ }^{1,2}$ Ala Alkharaan, ${ }^{1,2}$ Abdulrahman Alfawzan,,${ }^{1,2}$ Aysha Alshahrani, ${ }^{2,3}$ Emad Masuadi, ${ }^{1,2}$ Awad Alshahrani ${ }^{1,2,4}$

ABSTRACT: Objectives: This study aimed to explore Saudi Arabian medical students' perceptions of patient safety. Methods: A cross-sectional descriptive study was conducted in the College of Medicine, King Saud Bin Abdulaziz University for Health Sciences, Riyadh, Saudi Arabia, in September 2019. The Attitudes to Patient Safety Questionnaire (APSQ III) was used to explore undergraduate medical students' attitudes towards and knowledge of PS. The main outcomes measured were the APSQ III's nine domains. Data were analysed using Statistical Package for the Social Sciences and students' attitudes were communicated as mean scores \pm standard deviations. Results: A total of 301 participants were included in this study (response rate: 85.75\%). Six domains reflected a positive attitude while three domains showed a neutral attitude. The domain of 'team functioning' had the highest mean score (5.8) followed by 'working hours as a cause of error' (5.6) and 'error inevitability' (5.4). There was a significant difference between gender in the domain 'patient involvement in reducing error' $(P=0.012)$ and 'importance of patient safety (PS) in the curriculum' $(P=0.001)$. In addition, the 'importance of PS in the curriculum' domain was significantly different across different age groups $(P=0.039)$. Conclusion: Medical students were highly interested in PS and recommended implementing a comprehensive undergraduate PS programme to fulfil their educational needs.

Keywords: Undergraduate Medical Education; Patient Safety; Medical Errors; Attitude; Medical Students; Medical Ethics; Patient Care; Saudi Arabia.

\footnotetext{
Advances in KNOWLedge

The presence of receptive learning environment is one of the most important determinants to establish a patient safety programme of the best outcomes.

The confidence of medical students to report an error is negatively implicated by the unprofessional role modeling within a dominant clinical hierarchy.

Male physicians prefer to follow a paternalistic relationship with patients compared to their female encounters.
}

\section{Application to Patient Care}

Undergraduate education serves an important role in maintaining patient safety and the introduction of an undergraduate patient safety programmes in Saudi universities would highly benefit the quality of future healthcare.

Pateint safety curriculam should provide practical guidance for healthcare providers to achieve better doctor-patient relationships and outstanding problem solving skills and minimise unwanted complications if adverse events occur.

Medical students' educational needs, course timing and differences in exposure to hospital environments should be considered when designing an undergraduate medical curriculum in patient safety.

W ITH DEVELOPMENTS IN SCIENCE AND TECHnology, the modern healthcare system has hugely improved the quality of medical care. However, medical errors have become a serious issue globally over the past decades. ${ }^{1,2}$ In 1999, the USA's Institute of Medicine Committee on Quality of Health Care in America reported that 98,000 hospitalised patients die annually due to medical error, exceeding the number of deaths caused by motor vehicle accidents, breast cancer or AIDS. ${ }^{1}$ In response to the striking number of deaths caused by medical error, an Institute of Medicine report aimed to raise attention around patient safety and the need for such an important discipline as a part of medical education.1 Between 1999-2013, preventable deaths caused by adverse medical events exceeded 250,000 in the USA and medical error now ranks as the third most common cause of death in the USA. ${ }^{2}$

Medical errors are becoming a major consumer of healthcare system resources, causing burdens associated with both economics and death. In 2008, medical errors cost the USA $\$ 19.5$ billion and $\$ 1.4$ billion were ascribed to increased mortality rate and the resulting loss of productivity, which is estimated to equate to 10 million work days lost due to missed work caused by disability claims. ${ }^{3}$ In Saudi Arabia, the rate of medical errors has been escalating. The total number of medical error claims in Saudi Arabia in 1999 were 440 and increased to 1,356 cases in $2008 .^{4}$ In 2016, a local study reported that financial compensation due 
to medical errors claims was rising in Saudi Arabia, with $45.78 \%$ of death cases compensated with between USD $\$ 26,000-130,000$ and $53.65 \%$ of disability cases compensated with between USD \$260-13,000. ${ }^{5}$

One of the most-known solutions to reduce medical error and improve patient care is implementing a patient safety system in hospitals through a critical incident reporting system. These systems provide clear guidelines on how and what to report and enhances the availability of valuable feedback through local meetings, paper contributions or email alerts. ${ }^{6}$ Patient safety systems encourage education and training on patient safety topics by providing useful tools such as checklists, structured language, or set of phrases the team has agreed to use in operating rooms. ${ }^{7}$ In 2009, the World Health Organization (WHO) stated the importance of incorporating patient safety into medical curricula in order to orient medical students to the need for patient safety. ${ }^{8}$ The WHO's Patient Safety Curriculum Guide for Medical Schools contains 11 evidencebased chapters on patient safety and demonstrates that traditional medical schools teach and assess student performance in three main areas: medical knowledge, technical skills and clinical decision-making. ${ }^{8}$ Newer medical education teaches and evaluates these three traditional technical skills in addition to non-technical skills: communication, risk awareness and management, teamwork and leadership. ${ }^{8}$

Several measurements have recently been used to assess medical errors and many actions have been taken to enhance patient safety globally. The widespread culture among physicians and their attitudes towards patient safety, remain important determinant factors; however, these attitudes may present an obstacle. ${ }^{9}$ Therefore, many studies have recommended instituting patient safety educational programmes in hospitals for physicians and other healthcare providers. ${ }^{10}$ Previous questionnaires, such as the Madigosky et al. questionnaire and the Attitudes to Patient Safety Questionnaire III (APSQ-III), have been used to determine medical students' awareness and knowledge of and attitudes towards patient safety. ${ }^{9,11}$ These tools can potentially help medical educators plan and implement classroom experiences to fulfil students' needs around patient safety. ${ }^{9,11}$

Because healthcare systems worldwide are undergoing transformational development in the quality of patient care, it is essential to ensure satisfactory education in patient safety for future doctors. It is also important to provide baseline characteristics of students' perceptions and needs regarding patient safety to serve as useful data when designing an appropriate curriculum for undergraduate medical students. There is a shortage of studies on this topic in
Saudi Arabia. Therefore, this study aimed to explore the perceptions and attitudes of undergraduate medical students towards patient safety education.

\section{Methods}

This cross-sectional descriptive study was conducted in September 2019 at the College of Medicine, King Saud Bin Abdelaziz University for Health Sciences (KSAUHS), which is located in Riyadh, the capital of Saudi Arabia. Participants were undergraduate medical students from first to fourth year programmes. The academic programme at KSAUHS's College of Medicine is a total of four years. The first and second years are the pre-clinical phase while the third and fourth years are devoted to clinical rounds. Students from other colleges were excluded. Based on an estimated total population of 4,000 medical students in Riyadh, the necessary sample size was calculated using a Raosoft calculator (Raosoft Inc., Seattle, Washington, USA) at a 95\% confidence level and a $5 \%$ margin of error. Assuming a 50\% response rate, 351 participants were recruited with non-probability convenience sampling.

At KSAUHS, all students must attend a problembased learning (PBL) session twice weekly. In this PBL session, students are usually divided into groups of 10

Table 1: Characteristics of Saudi Arabian undergraduate medical students included in this study $(\mathrm{N}=301)$

$\begin{array}{lc}\text { Characteristic } & \mathbf{n}(\%) \\ \text { Gender } & \\ \text { Male } & 249(82.72) \\ \text { Female } & 52(17.28) \\ \text { Academic year } & \\ \text { First } & 70(23.26) \\ \text { Second } & 80(26.58) \\ \text { Third } & 95(31.56) \\ \text { Fourth } & 56(18.60) \\ \text { Age in years } & \\ 20 & 24(7.97) \\ 21 & 75(24.92) \\ 22 & 77(25.58) \\ 23 & 64(21.26) \\ 24 & 23(7.64) \\ \geq 25 & 38(12.62) \\ \text { Mean age in years } \pm \text { SD } & 22 \pm 1.94\end{array}$

$S D=$ standard deviation 
Table 2: Attitude of Saudi Arabian undergraduate medical students towards patient safety using the Attitudes to Patient Safety Questionnaire

\section{Items*}

1. My training is preparing me to understand the causes of medical errors.

2. I have a good understanding of patient safety issues as a result of my undergraduate medical training.

3. My training is preparing me to prevent medical errors.

4. I would feel comfortable reporting any errors I had made, no matter how serious the outcome had been for the patient.

5. I would feel comfortable reporting any errors other people had made, no matter how serious the outcome had been for the patient.

6. I am confident I could talk openly to my supervisor about an error I had made if it had resulted in potential or actual harm to my patient.

7. Shorter shifts for doctors will reduce medical errors.

8. By not taking regular breaks during shifts, doctors are at an increased risk of making errors.

9. The number of hours doctors work increases the likelihood of making medical errors.

10. Even the most experienced and competent doctors make errors

11. A true professional does not make mistakes or errors. ${ }^{\dagger}$

12. Human error is inevitable.

13. Most medical errors result from careless nurses. ${ }^{\dagger}$

14. If people paid more attention at work, medical errors would be avoided. ${ }^{\dagger}$

15. Most medical errors result from careless doctors. ${ }^{\dagger}$

16. Medical errors are a sign of incompetence. ${ }^{\dagger}$

17. It is not necessary to report errors which do not result in adverse outcomes for the patient. ${ }^{\dagger}$

18. Doctors have a responsibility to disclose errors to patients only if they result in patient harm. ${ }^{\dagger}$

19. All medical errors should be reported.

20. Better multi-disciplinary teamwork will reduce medical errors.

21. Teaching teamwork skills will reduce medical errors.

22. Patients have an important role in preventing medical errors.

23. Encouraging patients to be more involved in their care can help to reduce the risk of medical errors occurring.

24. Teaching students about patient safety should be an important priority in medical students training.

25. Patient safety issues cannot be taught and can only be learned by clinical experience when qualified. ${ }^{\dagger}$

26. Learning about patient safety issues before I qualify will enable me to become a more effective doctor.
Mean score \pm SD

$4.45 \pm 1.53$

$4.62 \pm 1.63$

$4.75 \pm 1.77$

$4.29 \pm 1.81$

$4.99 \pm 1.76$

$5.54 \pm 1.63$

$5.63 \pm 1.46$

$5.75 \pm 1.41$

$5.79 \pm 1.41$

$2.43 \pm 1.66$

$4.97 \pm 1.80$

$3.04 \pm 1.69$

$4.87 \pm 1.68$

$3.63 \pm 1.71$

$3.52 \pm 1.56$

$2.71 \pm 1.78$

$3.70 \pm 2.04$

$5.66 \pm 1.64$

$5.88 \pm 1.51$

$5.79 \pm 1.49$

$4.88 \pm 1.61$

$5.25 \pm 1.53$

$5.67 \pm 1.57$

$3.47 \pm 1.79$

$5.41 \pm 1.60$

"Graded according to a 7-point Likert scale ( $7=$ strongly agree to $1=$ strongly disagree). A score of $>4$ indicates a positive attitude, a negative attitude is indicated by a score $<4$ and a neutral attitude by a score of $4 .{ }^{\dagger}$ Reverse coded items.

to discuss a medical case for 90 minutes. After this PBL session ended, a self-administered questionnaire was given to all groups. Only students who agreed to participate stayed after the PBL session to complete the questionnaire. Data were collected between 17-31 September 2019 by research team members who were also medical students.

This study provided baseline characteristics of students' perceptions of and attitudes towards patient safety education using the APSQ-III. The questionnaire was adapted and validated by Carruthers et al., and permission to utilise the APSQ-III was obtained from the original author via email. ${ }^{9}$ Demographic data collected included age, gender, academic year or batch group and respondent's college. Measured outcomes were student perceptions and attitudes; these were assessed through 26 questions in nine domains: 'PS training received' (items 1-3); 'error reporting confidence' (items 4-6); 'working hours as a cause of error' (items 7-9); 'error inevitability' (items 10-12); 
Table 3: Saudi Arabian undergraduate medical students' attitudes towards patient safety according to the nine domains of the Attitudes to Patient Safety Questionnaire

\begin{tabular}{|c|c|c|c|c|c|c|c|c|c|c|c|}
\hline \multirow[t]{2}{*}{ Domain } & \multirow{2}{*}{$\begin{array}{c}\text { Mean } \\
\text { overall } \\
\text { score } \pm \text { SD }\end{array}$} & \multicolumn{3}{|c|}{ Gender } & \multicolumn{7}{|c|}{ Age in years } \\
\hline & & Male & Female & $\underset{\text { value }^{*}}{P}$ & 20 & 21 & 22 & 23 & 24 & $\geq 25$ & $\begin{array}{c}P \\
\text { value }^{\dagger}\end{array}$ \\
\hline 1. PS training received & $4.6 \pm 1.33$ & 4.55 & 4.55 & 0.989 & 4.18 & 4.72 & 4.49 & 4.51 & 4.93 & 4.43 & 0.360 \\
\hline $\begin{array}{l}\text { 2. Error reporting } \\
\text { confidence }\end{array}$ & $4.7 \pm 1.56$ & 4.65 & 4.67 & 0.939 & 4.97 & 4.86 & 4.81 & 4.43 & 4.39 & 4.38 & 0.278 \\
\hline $\begin{array}{l}\text { 3. Working hours as a } \\
\text { cause of error }\end{array}$ & $5.6 \pm 1.27$ & 5.60 & 5.65 & 0.763 & 5.71 & 5.79 & 5.76 & 5.40 & 5.39 & 5.65 & 0.423 \\
\hline 4. Error inevitability & $5.4 \pm 1.22$ & 5.49 & 5.43 & 0.746 & 5.14 & 5.44 & 5.66 & 5.22 & 5.45 & 5.54 & 0.276 \\
\hline $\begin{array}{l}\text { 5. Professional } \\
\text { incompetence as a cause } \\
\text { of error }\end{array}$ & $4.2 \pm 1.18$ & 4.42 & 4.19 & 0.207 & 4.31 & 4.26 & 4.08 & 4.04 & 4.34 & 4.70 & 0.092 \\
\hline 7. Team functioning & $5.8 \pm 1.36$ & 6.13 & 5.77 & 0.088 & 5.33 & 5.77 & 5.91 & 5.70 & 6.24 & 6.11 & 0.162 \\
\hline $\begin{array}{l}\text { 8. Patient involvement in } \\
\text { reducing error }\end{array}$ & $5.1 \pm 1.32$ & 5.48 & 4.98 & 0.012 & 4.94 & 5.07 & 4.86 & 4.98 & 5.24 & 5.61 & 0.101 \\
\hline $\begin{array}{l}\text { 9. Importance of PS in the } \\
\text { curriculum }\end{array}$ & $5.2 \pm 1.20$ & 5.09 & 5.69 & 0.001 & 5.31 & 5.07 & 5.11 & 4.99 & 5.52 & 5.70 & 0.039 \\
\hline
\end{tabular}

$S D=$ standard deviation; $P S=$ patient safety .

*Calculated using Student's t-test. ${ }^{\dagger}$ Calculated using ANOVA.

'professional incompetence as a cause of error' (items 13-16); 'disclosure responsibility' (items 17-19); 'team functioning' (items 20 and 21); 'patient involvement in reducing error' (items 22 and 23); and 'importance of PS in the curriculum' (items 24-26). ${ }^{15} \mathrm{~A}$ 7-point Likert scale ( 1 = strongly disagree, 2 = disagree, 3 = somewhat disagree, $4=$ neutral, $5=$ somewhat agree, $6=$ agree and 7 = strongly agree) was used to measure students' perceptions and attitudes. The instrument's creator reversed the coding on items $11,13,14,15,16,17,18$ and 25 , where the highest score is one and seven is the lowest because those items indicate negative beliefs. Reverse coding was used for those items to obtain the mean score of each domain.

An Excel (Microsoft, Corp., Redmond, Washington, USA) sheet was used for data entry, and Statistical Package for the Social Sciences (SPSS), Version 21 (IBM, Corp., Armonk, New York, USA) was used for data analysis. Students were grouped based on gender, academic year and age. Descriptive statistics were used to present demographic data in frequency and percentages. Students' perceptions of each statement in the APSQ-III were displayed in mean \pm standard deviation. The perception score was considered positive if the response range was from $5-7$ or negative if the score was from $1-3$. In the case of reverse coded questions, responses were considered positive if the response range was from 1-3. A mean score of 4 in both cases was considered neutral. Students' perceptions and attitudes were also analysed

according to the corresponding nine domains of the APSQ-III. One-way analysis of variance was used to compare the mean scores of the students' perceptions between the five age categories. To note differences by gender, a Student's t-test was used to compare the mean scores of students' perceptions and attitudes. The level of statistical significance was set at $P<0.05$.

Informed written consent was given by all participants and questionnaire completion was voluntary and anonymous. The study was approved by King Abdullah International Medical Research Center, and the data were collected after gaining approval from the university's Institutional Review Board (RC19/065/R). Confidentiality and anonymity were maintained during and after data collection.

\section{Results}

A total of 301 participants were included in this study (response rate: $85.75 \%$ ). There was a male predominance $(\mathrm{n}=249 ; 82.72 \%)$ and most participants were in their third year $(\mathrm{n}=95 ; 31.56 \%)$ and 22 years old ( $\mathrm{n}=77 ; 25.58 \%$ ) [Table 1 ]. Interestingly, no items were perceived with a negative attitude and 12 items revealed a positive attitude while 14 items showed a neutral attitude [Table 2]. Most participants (82.40\%) agreed that 'Better multi-disciplinary teamwork will reduce medical errors' (mean $=5.88$ ). Most participants supported the perception that 'teaching teamwork 
skills will reduce medical errors' and 'even the most experienced and competent doctors make errors' (mean $=5.79$ each). In regard to the nine domains, 'team functioning' had the highest score (mean $=5.8$ ) followed by 'working hours as a cause of error' (mean = 5.6) and 'error inevitability' (mean = 5.4) [Table 3]. There was a statistical difference between gender in two key domains: 'patient involvement in reducing error' $(P=0.012)$ and 'importance of PS in the curriculum' $(P=0.001)$. In addition, the perception of 'importance of PS in the curriculum' showed a significant difference $(P=0.039)$ in terms of participants' ages.

\section{Discussion}

In order to encourage quality patient care in Saudi Arabia, the present study explored medical students' perceptions of and attitudes towards PS. Medical students' interests in PS are highlighted and common misconceptions that need to be addressed when building an undergraduate curriculum in such a field are discussed below.

In the present study, 'team functioning' had the highest score among all domains. Similarly, a study conducted in Pakistan also showed team functioning to be the highest scored item. ${ }^{12}$ This similarity reflects the importance of team-based teaching in medical curricula and healthcare institutions. Communication and collaboration skills as well as establishing a teamwork-focused culture are essential factors in PS and, hence, the quality of healthcare. ${ }^{13}$ Interestingly, the positive attitudes of medical students towards team functioning showed a nearly escalating pattern according to participants' ages. Heavy workloads of more than 40 hours a week and long overtime shifts of more than 12.5 hours per shift among healthcare providers were significantly associated with an increased risk of medical error $(P$ value $=0.0001$ and 0.005 , respectively). ${ }^{14}$ Similar to previous findings reported in the literature, medical students who participated in the current study strongly agreed that human error is inevitable and working hours are potential causes of medical errors. ${ }^{12,13,15}$

Traditional educational institutions assume that PS education is gained through clinical experience rather than educational knowledge-based courses. ${ }^{16}$ Nevertheless, the inclusion of PS education in undergraduate medical curricula would further strengthen practitioners' abilities to identifyand deal with situations threatening PS. ${ }^{2,3}$ Undergraduate students in different countries have supported such a suggestion..$^{12,13,15}$ In the present study, the attitudes of students positively $(5.2 \pm 1.20)$ supported implementing a knowledge- based programme designed for undergraduate medical students. The reported need of medical students to receive PS education could be explained by the absence of comprehensive undergraduate educational courses in medical schools. In Saudi Arabia, various academic institutions, that target all healthcare providers, provide selective courses, short lectures and annual meetings concerning topics related to PS. However, an undergraduate curriculum in PS is not mandatory for completing undergraduate medical education in Saudi universities and has been developed informally through bedside teaching in the clinical years with minimal exposure to PS-related terminology. Bedside teaching, where trainees interact with patients under the supervision of a clinician, remains an essential educational tool especially for junior doctors to promote and accelerate clinical maturity. ${ }^{17}$ However, reliance only on bedside teaching model, which is often provided in a heterogeneous manner, is challenging to reach a standardised consistent PS education. Standardisation of the required knowledge and skills would result in achieving a satisfactory level of knowledge related to PS. A lack of available faculty, time and funding, accreditation requirements and differing hospital environments diversifies students' exposure to bedside teaching across various medical colleges. ${ }^{16,17}$

Undergraduate students in the current study showed a uniquely positive attitude towards teaching PS. A receptive learning environment is key in establishing a curriculum that supports best outcomes. ${ }^{18}$ Nursing students, who are thought to be more familiar with PS topics, think that there is insufficient time spent learning about PS and quality of patient care. ${ }^{19}$ This finding is relative to undergraduate courses that extensively discuss pathophysiology, management and prognosis of medical diseases. ${ }^{19}$ Although the average score of the domain 'importance of PS in the curriculum' was high for both males and females, there was a significant difference between the genders $(P=0.001)$; a significant difference was also noted in terms of students' ages $(P=0.039)$. The average score of this domain increased gradually with the increase in academic years. This finding might indicate a higher need for PS education, particularly for senior students. In the current study, most participants over 25 years old had previous healthcare experience and had joined medical school after completing a bachelor's degree in other healthcare specialties.

The factor of receiving PS training had the second lowest score in the current study, which may indicate a knowledge gap in understanding PS issues and the ability to prevent medical error. Madigosky et al. investigated the effects of introducing a PS and medical 
fallibility course for undergraduate medical students by evaluating students' responses to a knowledge, skills and attitudes questionnaire before and after course completion. ${ }^{11}$ A total of $72 \%$ of students responded that the course fulfilled their medical objectives, including recognising human inevitability, addressing factors related to medical errors, learning and improving skills for error disclosure and providing a guide for ideal communication within healthcare environments. The introduction of a PS course has been identified as beneficial and has been highly recommended for undergraduate medical education.,11-13,15,19 In the Asser region of Saudi Arabia, the APSQ-III was given to primary care physicians; one-fifth were unsatisfied with their training and $70 \%$ had not received any training, although most participants demonstrated a positive attitude towards PS education..$^{20}$ These results show the necessity of prioritising PS education to undergraduate medical students.

In the current study, students showed positive attitudes towards disclosure responsibility. The level of error reporting confidence was shown to be good, to a limited extent, among the current participants. The willingness to report error declines in students as they achieve higher academic levels. This decline in error reporting confidence might be caused by a fear of speaking-up within a dominant clinical hierarchy. In Germany, first-year students have been found to have higher willpower to disclose medical errors. ${ }^{13}$ The exposure of medical students to negative role modeling or toxic bedside teaching during their clinical experience might promote the development of unprofessional behaviours such as neglecting important ethical concepts related to patient autonomy and avoiding disclosing adverse medical events. ${ }^{13}$

The effect of the 'hidden curriculum', which dominates medical education, affects medical students as they develop ethical and professional identities as junior doctors. Educators and clinicians must take into consideration the harmful influence of the "hidden curriculum' and instead promote open discussion among team members to convey any concerns related to PS. ${ }^{21}$ To support the practice of error disclosure, healthcare providers should avoid blaming each other and instead encourage an interdisciplinary clinical culture that understands limited human capability and prioritises outstanding patient care through proper communication between team members. ${ }^{13,22-24}$ In fact, the practice of error disclosure is subjected to cultural considerations because unnecessary disclosure, if no clear adverse events were encountered, might result in patient distress. ${ }^{22}$ Hammami et al. surveyed patients visiting outpatient clinic in Riyadh and found that 95\% of participants prefer to be informed if major, minor or near-miss medical errors had occurred..$^{23}$ From an Islamic point of view, error must be disclosed and forgiveness should be sought. ${ }^{23}$

'Professional incompetence as a cause of error' scored the lowest among all domains. Although most of students had positive perceptions regarding error inevitability, the misconception that medical errors are due to underlying individual failures should be addressed when implementing patient safety curricula in the future. The attitude towards patient involvement in reducing medical errors significantly differs between genders $(P=0.012)$. In terms of communication, Roter et al. reported that female physicians were more likely to spend greater time in patient-centred talk than their male colleagues, with an average of two minutes or 10\% of the time per visit. ${ }^{25}$ Löffler-Stastka et al. stated that female students were more likely to express empathy, address psychosocial concerns while taking medical history and spend greater time communicating with patients. ${ }^{26}$ On the other hand, Löffler-Stastka et al. estimated that one-third of male physicians preferred to follow a paternalistic relationship with patients, whereas female physicians were more interested in patient-centred communication. ${ }^{26}$

Using a validated and reliable tool to assess students' thinking, including students from four academic years and recruiting a larger number of participants relative to previous studies, are strong features of the present study. However, this study has some limitations. First, it could not investigate the effectiveness of introducing an educational programme in patient safety for medical students. Rather, this study provides baseline data to help design a proper undergraduate curriculum in the future. Second, the response rate in the current study was lower than expected, especially from female students; this low response rate was in part due to the study's convenience sampling, which was a result of students' absence during the data collection and a higher percentage of male students than female students in the College of Medicine. In order to determine the effectiveness of PS education on the quality of patient care, a longitudinal evaluation should be done during and after introducing a PS education programme.

\section{Conclusion}

The APSQ-III tool revealed a positive attitude among Saudi undergraduate medical students towards PS. A knowledge gap still exists in major aspects of PS such as the role of professional incompetence in causing errors and the confidence to report medical errors. The current status of undergraduate education on PS has also been reported as inadequate. Clinical-year 
students are likely to benefit more from introducing undergraduate courses in PS. Educational institutions should consider implementing a comprehensive undergraduate educational programme on PS to meet the needs of future doctors. Further longitudinal studies are required to evaluate the effectiveness of such an intervention.

\section{CONFLICT OF INTEREST}

The authors declare no conflicts of interest.

\section{FUNDING}

No funding was received for this study.

\section{References}

1. Institute of Medicine (US) Committee on Quality of Health Care in America, Kohn LT, Corrigan JM, Donaldson MS, Eds. To Err is Human: Building a Safer Health System. Washington, DC, USA: National Academies Press, 2000.

2. Makary MA, Daniel M. Medical error-The third leading cause of death in the US. BMJ 2016; 353:i2139. https://doi. org/10.1136/bmj.i2139.

3. Andel C, Davidow SL, Hollander M, Moreno DA. The economics of health care quality and medical errors. J Health Care Finance 2012; 39:39-50

4. Al-Saeed AH. Medical liability litigation in Saudi Arabia Saudi J Anaesth 2010; 4:122-6. https://doi.org/10.4103/1658354X.71133.

5. Alshammari MH, Mital DP. Medical errors in Saudi Arabia: Understanding the pattern and associated financial cost. Int J Med Engin Inform 2016; 8:41-8. https://doi.org/10.1504/ IJMEI.2016.073652.

6. Health Quality Ontario. Patient safety learning systems: A systematic review and qualitative synthesis. Ont Health Technol Assess Ser 2017; 17:1-23.

7. Oppikofer C, Schwappach D. The role of checklists and human factors for improved patient safety in plastic surgery. Plast Reconstr Surg 2017; 140:812e-17e. https://doi.org/10.1097/ PRS.0000000000003892.

8. World Health Organization. WHO patient safety curriculum guide for medical schools. From: https://who.int/iris/ handle/10665/44091 Accessed: Jun 2020.

9. Carruthers S, Lawton R, Sandars J, Howe A, Perry M. Attitudes to patient safety amongst medical students and tutors: Developing a reliable and valid measure. Med Teach 2009; 31:e370-6. https://doi.org/10.1080/01421590802650142.

10. Hindle D, Braithwaite J, Iedema R. The Centre for Clinical Governance Research, Patient Safety Research: A review of the technical literature. Sydney, Australia: University of New South Wales; 2005.

11. Madigosky WS, Headrick LA, Nelson K, Cox KR, Anderson T. Changing and sustaining medical students' knowledge, skills, and attitudes about patient safety and medical fallibility. Acad Med 2006; 81:94-101. https://doi.org/10.1097/00001888-20060 1000-00022.
12. Kamran R, Bari A, Khan RA, Al-Eraky M. Patient safety awareness among undergraduate medical students in Pakistani Medical School. Pak J Med Sci 2018; 34:305-9. https://doi. org/10.12669/pjms.342.14563.

13. Kiesewetter J, Kager M, Lux R, Zwissler B, Fischer MR, Dietz I. German undergraduate medical students' attitudes and needs regarding medical errors and patient safety--A national survey in Germany. Med Teach 2014; 36:505-10. https://doi.org/10.31 09/0142159X.2014.891008.

14. Rogers AE, Hwang WT, Scott LD, Aiken LH, Dinges DF. The working hours of hospital staff nurses and patient safety. Health Aff (Millwood) 2004; 23:202-12. https://doi.org/10.1377/hlt haff.23.4.202.

15. Leung GK, Ang SB, Lau TC, Neo HJ, Patil NG, Ti LK. Patient safety culture among medical students in Singapore and Hong Kong. Singapore Med J 2013; 54:501-5. https://doi.org/10.1162 2/smedj.2013172.

16. Wu AW, Busch IM. Patient safety: A new basic science for professional education. GMS J Med Educ 2019; 36:Doc21. https://doi.org/10.3205/zma001229

17. Peters $\mathrm{M}$, Ten Cate $\mathrm{O}$. Bedside teaching in medical education: A literature review. Perspect Med Educ 2014; 3:76-88. https:// doi.org/10.1007/s40037-013-0083-y.

18. Bowman C, Neeman N, Sehgal NL. Enculturation of unsafe attitudes and behaviors: Student perceptions of safety culture. Acad Med 2013; 88:802-10. https://doi.org/10.1097/ACM.0b 013e31828fd4f4.

19. Vaismoradi M, Salsali M, Marck P. Patient safety: Nursing students' perspectives and the role of nursing education to provide safe care. Int Nurs Rev 2011; 58:434-42. https://doi. org/10.1111/j.1466-7657.2011.00882.x.

20. Al-Khaldi YM. Attitude of primary care physicians toward patient safety in Aseer region, Saudi Arabia. J Family Community Med 2013; 20:153-8. https://doi.org/10.4103/2230-8229.121976.

21. Lehmann LS, Sulmasy LS, Desai S; ACP Ethics, Professionalism and Human Rights Committee. Hidden curricula, ethics, and professionalism: Optimizing clinical learning environments in becoming and being a physician: A position paper of the American College of Physicians. Ann Intern Med 2018; 168:506-8. https://doi.org/10.7326/M17-2058.

22. Ethics Committee of the American Society for Reproductive Medicine. Disclosure of medical errors involving gametes and embryos. Fertil Steril 2006; 86:513-15. https://doi.org/10.10 16/j.fertnstert.2006.07.1448.

23. Hammami MM, Attalah S, Al Qadire M. Which medical error to disclose to patients and by whom? Public preference and perceptions of norm and current practice. BMC Med Ethics 2010; 11:17. https://doi.org/10.1186/1472-6939-11-17.

24. Almaramhy H, Al-Shobaili H, El-Hadary K, Dandash K. Knowledge and attitude towards patient safety among a group of undergraduate medical students in Saudi Arabia. Int J Health Sci (Qassim) 2011; 5:59-67.

25. Roter DL, Hall JA, Aoki Y. Physician gender effects in medical communication: A meta-analytic review. JAMA 2002; 288:756-64. https://doi.org/10.1001/jama.288.6.756.

26. Löffler-Stastka H, Seitz T, Billeth S, Pastner B, Preusche I, Seidman C. Significance of gender in the attitude towards doctorpatient communication in medical students and physicians. Wien Klin Wochenschr 2016; 128:663-8. https://doi.org/10.1007/ s00508-016-1054-1. 